\title{
Correction to: A New Approach for Tuned Clustering Analysis
}

\author{
Roni Ben Ishay, Maya Herman, and Chaim Yosefy
}

\section{Correction to:}

Chapter "A New Approach for Tuned Clustering Analysis" in: P. Perner (Ed.): Machine Learning and Data Mining in Pattern Recognition, LNCS 10934, https://doi.org/10.1007/978-3-319-96136-1_34

The original version of this chapter contained an error in the third author's name. The spelling of Chaim Yosefy's name was incorrect in the header of the paper. The author name has been corrected. 